\title{
Excipient Stability: a Critical Aspect in Stability of Pharmaceuticals
}

\author{
S. Narasimha Murthy ${ }^{1,2}$ and Michael A. Repka ${ }^{1}$
}

Received 2 October 2017; accepted 5 October 2017; published online 21 November 2017

Pharmaceutical dosage forms are generally incorporated with several functional and organoleptic excipients. The term stability of a pharmaceutical dosage form implies the physical and chemical integrity of the active pharmaceutical ingredient (API), the excipients and intactness of the packaging [1]. Excipients play an important role in determining the quality and performance attributes of pharmaceutical dosage forms [2]. Additionally, certain excipients also influence the sensorial characteristics such as appearance, odor, taste, and texture of the products as well. The stabilizers preserve the API from getting affected by micro- and macro-environmental factors [3]. In some dosage forms, the instability may also be induced due to extreme processing conditions like high temperature or relative humidity [4].

Any unintended change in the inherent nature and physicochemical characteristics of pharmaceutical excipients could lead to potential instabilities in the formulation that could disrupt the quality and performance attributes of the product. Interaction among the excipients is also a potential concern, as it could lead to significant incompatibilities in the product [5]. Therefore, selection of appropriate excipients with good mutual compatibility is crucial in developing a quality product that sustains its desired properties throughout the shelf life period. Particularly, in case of preservatives, the loss of antibacterial activity due to binding to polymers and surfactants in the formulation or to the packaging materials could lead to microbial burden in the product. The growth of pathological microorganisms would be rather a greater concern [6].

Therefore, a thorough preformulation research is a requirement prior to development of pharmaceutical

\footnotetext{
${ }^{1}$ Department of Pharmaceutics and Drug Delivery, School of Pharmacy, The University of Mississippi, University, MS 38677, USA.

${ }^{2}$ To whom correspondence should be addressed. (e-mail: murthy@olemiss.edu)
}

dosage forms. There are a variety of tools and methods available to investigate the instabilities and interactions associated with pharmaceutical excipients. The spectroscopic techniques, chromatographic and physiochemical analysis of excipients in its neat form as well as in combination with API and other excipients could aid in making appropriate choices of excipients during product development [7]. Besides understanding the interaction and change in physicochemical nature of excipient compositions, the research should also focus on potential approaches to overcome the instabilities.

The focus of the present theme is to include review articles emphasizing the excipient stability. While some articles review the excipients directly, the other articles review stability issues emanating due to poor choice of excipients. The articles also include discussions on various tools available to investigate any excipient related incompatibilities, interactions, and degradations. Further, the potential approaches to surmount such undesirable changes in the pharmaceutical products are also reviewed.

\section{REFERENCES}

1. USP HS. United States Pharmacopeia-National Formulary, USP 35-NF 30. Rockville.

2. Pifferi G, Santoro P, Pedrani M. Quality and functionality of excipients. Farmaco. 1999;54(1):1-4.

3. Pawar S, Kumar A. Issues in the formulation of drugs for oral use in children. Pediatr Drugs. 2002;4(6):371-9.

4. Kumar N, Jha A. Temperature excursion management: a novel approach of quality system in pharmaceutical industry. Saudi Pharmaceutical Journal. 2017;25(2):176-83.

5. Narang AS, Desai D, Badawy S. Impact of excipient interactions on solid dosage form stability. Pharm Res. 2012;29(10):2660-83.

6. Hanlon G, Hodges NA. Essential microbiology for pharmacy and pharmaceutical science. Hoboken: Wiley; 2012.

7. Mohamed AI, Abd-Motagaly AM, Ahmed OA, Amin S, Mohamed Ali AI. Investigation of drug-polymer compatibility using chemometric-assisted UV-spectrophotometry. Pharmaceutics. 2017;9(1):7. 